Case Report

\title{
A Rare Case of Benign Type RIIIA Single Coronary Artery Anomaly
}

\author{
Mahesh Deshpande', Vinod Shetkar', Kavita Upadhyay ${ }^{3}$, Renu Chauhan ${ }^{4}$ \\ ${ }^{1}$ DNB Cardiology, Intervention Cardiologist, Dr. Hedgewar Hospital, Aurangabad, Maharashtra, India. \\ ${ }^{2}$ M.Ch-CVTS MRCS-Edinburgh U.K., Dr. Hedgewar Hospital, Aurangabad, Maharashtra, India. \\ ${ }^{3}$ Resident, D.N.B. General Medicine, Dr. Hedgewar Hospital, Aurangabad, Maharashtra, India. \\ ${ }^{4}$ D.A., Cardiac Anesthetist, Dr. Hedgewar Hospital, Aurangabad, Maharashtra, India. \\ DOI: https://doi.org/10.24321/2349.7181.202003
}

\section{I $\quad \mathbf{N} \quad \mathbf{F} \quad \mathbf{O}$}

\author{
Corresponding Author: \\ Mahesh Deshpande, Intervention Cardiologist, Dr. \\ Hedgewar Hospital, Aurangabad, Maharashtra, \\ India. \\ E-mail Id: \\ dr.deshpande.mahesh@gmail.com \\ Orcid Id: \\ https://orcid.org/0000-0002-3188-1870 \\ How to cite this article: \\ Deshpande M, Shetkar V, Upadhyay K, Chauhan \\ R. A Rare Case of Benign Type RIIIA Single \\ Coronary Artery Anomaly. J Adv Res Med 2020; \\ 7(1): 14-16. \\ Date of Submission: 2020-06-02 \\ Date of Acceptance: 2020-07-06
}

\section{$\begin{array}{llllllll}\mathbf{A} & \mathbf{B} & \mathbf{S} & \mathbf{T} & \mathbf{R} & \mathbf{A} & \mathbf{C} & \mathbf{T}\end{array}$}

Single Coronary Artery (SCA) is a rare anomaly with variable presentation, ranging from mild non-specific symptoms up to sudden cardiac death. A 60-year-old hypertensive female presented with acute coronary syndrome. Selective coronary angiography revealed Lipton's type RIIIA single coronary artery arising from Right sinus. Patient underwent CABGs and intraoperatively angiographic findings were confirmed. Despite having Type RIII, the LAD and LCX ran benign course, which is unusual.

Keywords: Absent left coronary artery; Single coronary artery anomaly, benign RIIIA variant

\section{Background}

Single Coronary Artery (SCA) in absence of congenital heart disease is an extremely rare anomaly. The prevalence of SCA in angiographic studies is in range of $0.024 \%$ to $1.85 \% .^{1-3}$ Modified Lipton's classification discussed them in detail. ${ }^{1}$ We hereby present a very rare case of SCA (Modified Lipton class R III A) where a common trunk arose from right coronary sinus branching into Left Anterior Descending (LAD), Left Circumflex (LCX) and Right Coronary Arteries (RCA). It is important to know the variations in anatomical course as some of the variants can cause sudden death while others run a benign course. Timely diagnosis can prevent deaths in malignant types by intervention.

\section{Case Report}

A 60-year-old hypertensive female presented with Non-ST Segment Elevation Myocardial Infarction (NSTEMI). The patient presented with complaints of typical angina associated with sweating for duration of approximately 48 hours. Historically, she was housewife with NYHA class II status but over last 2 days she progressed to uneasiness even at rest or with less than ordinary activity (NYHA IV). On admission, she had heart rate of $80 \mathrm{bpm}$, blood pressure of 130/90 $\mathrm{mmHg}$ and temperature of 36.6 ${ }^{\circ} \mathrm{C}$. Physical examination was unremarkable.

Electrocardiogram on day 1 showed ST segment 
depression in leads V2-V6, with and T wave inversion in leads II, III and avF. Cardiac biomarker, i.e., troponin I was found to be elevated significantly, i.e., 1063 pg/ml. Trans-thoracic echocardiogram revealed Regional Wall motion abnormality in right coronary artery territory and mild Left Ventricular (LV) Dysfunction (LV ejection fraction=45\%). Hematological investigations showed normal complete blood count and metabolic profile.

Diagnostic coronary angiography was done.

On day 2 , in diagnostic coronary angiography, LCA was found to be absent even after thorough probing and non-selective sinus injections (Figure 1). After selectively cannulating right coronary sinus (RCS); it was realized that patient had single coronary artery arising from RCS (Figure 2 and 3). The common trunk was very large and all three coronaries arising separately. LCX was the first branch, it had anterior course. It emerged on anterolateral wall with further branching into three major marginal branches; all having significant disease. LAD had a retro-aortic course, finally turning anteriorly from lateral side of pulmonary artery to emerge on anterolateral surface. It branched into diagonal (D1). Both native LAD and D1 had significant plaque with $70 \%$ stenosis.

RCA was continuation of SCA and ran normal course in atrio-ventricular groove. It had near total (99\%) long segment obstruction with grade $\mathrm{V}$ thrombus.

In view of triple vessel disease, she underwent CABGS on day 5. Intraoperatively, angiographic findings were confirmed, showing benign course of LAD and LCX. She was discharged in stable condition on day 12 (post-operative day 7). Informed consent was obtained from the patient and clearance was sought from Hospital Ethics Committee before reporting this rare case.

\section{Discussion}

When the entire coronary circulation is supplied through a lone coronary artery which may arise from either the left or right aortic sinus, it is called Single Coronary Artery (SCA) anomaly. Sometimes it may be associated with congenital heart disease. ${ }^{1}$

The separate origin of the LAD and LCX artery is the most common coronary artery anomaly. The second most common anomaly detected is origin of LCX arising from RCA. ${ }^{1}$ We report a rare case of isolated single coronary artery arising from right coronary cusp.

The original angiographic classification of SCA proposed by Lipton et al. ${ }^{4}$ was later modified by Yamanaka et al. ${ }^{5}$ (Table-1). As per this system, our patient would be classified as RIIIA.

Certain anomalous coronaries usually have a benign clinical course, such as RI, LI types. Meanwhile, the aberrant coronary artery courses between the outflow tracts such as R/LIIB or RIII, which makes them more prone to serious clinical complications. ${ }^{6}$ In the Lipton's RIII variant, LCX and LAD have been reported to cross either retroaortic, interarterial, anterior to pulmonary artery or course intramyocardially in previous literature. ${ }^{7}$ In our case, LAD and LCX cross anterior to pulmonary artery. During exercise, increased cardiac output leads to dilatation of great vessels leading to compression of aberrant coronaries along their varied course. ${ }^{6}$ Anginal symptoms and sudden cardiac death during exertion can be caused due to acute take-off angle of the anomalous vessel, slit-like orifice, and proximal intramural course. ${ }^{8,9}$

In our case the patient had a large common trunk arising from RCS. All the three coronaries had arisen separately from it and LCA was absent (Lipton RIIIA). The patient's LAD and LCX had a benign (non-intervascular) course. This may be the reason for event-free survival of the patient till 60 years and she presented with atherosclerotic coronary artery disease. She later underwent CABGs for her disease. There are very few case reports of RIIIA in medical literature. Panduranga et al \& Karahan et al reported similar RIII type SCA but with retroaortic LCX and anterior LAD. ${ }^{10,11}$ Shizukuda et al reported $\mathrm{R}$ IIIA type SCA with circumflex having intra-myocardial course. ${ }^{7}$ Our case was unique as it had no intervascular or intra-myocardial course and probably first case of RIIIA type SCA in India.

\section{Limitation}

CT coronary angiography is gold standard in evaluation of anomalous coronaries and it has a value in delineation of intramyocardial course. But in our case, CT coronary angiography was not feasible due to need for early revascularization for recurrent angina.

\section{Conclusion}

We report a rare case of isolated single coronary artery arising from RCS with absent LCA (Lipton RIIIA). The patient's LAD and LCX had a benign (non-interarterial) course. Thus, highlighting that not all RIII variants are life-threatening. Knowledge of such rare anomalies and course of coronaries is important for deciding revascularization strategy for patient. 


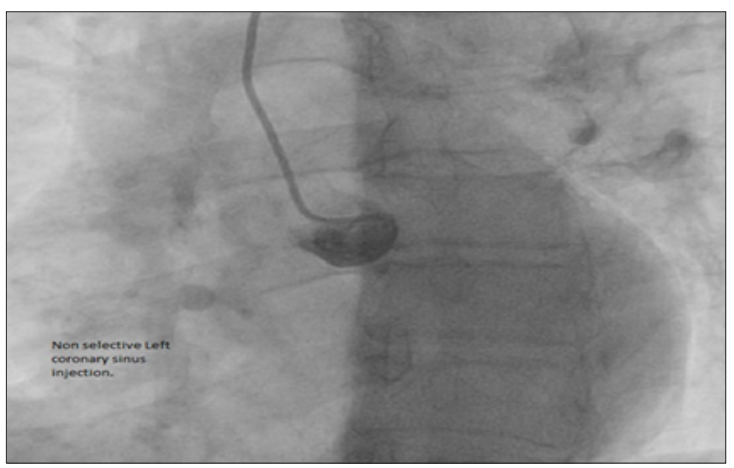

Image I.LAO 30. Non-selective left coronary sinus injection showing absent LCA

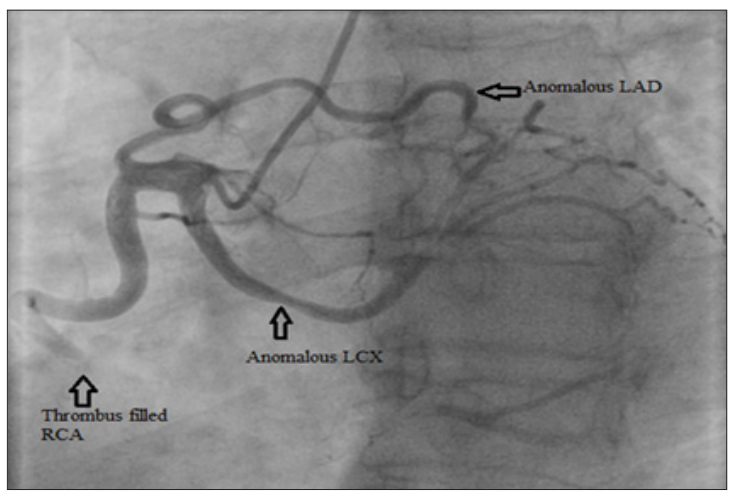

Image 2.LAO 30- shows LCX anterior course with trifurcation (arrow), RCA showing normal course with large thrombus filled occlusion (arrow)

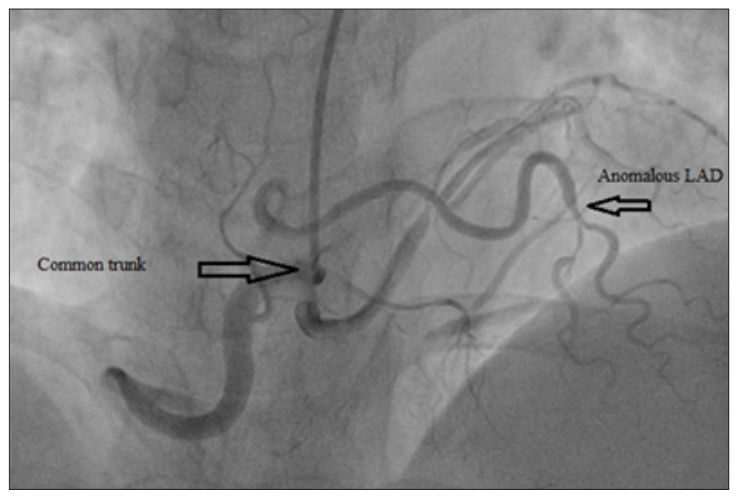

Image 3.AP-0 CRAN-30 VIEW: Large common trunk with retro-aortic course of LAD. After turning anteriorly there was significant LAD and DI disease (arrow)

Table I.Modified Lipton's classification of single coronary artery (I)

\begin{tabular}{|c|c|c|}
\hline Ostial location & $\begin{array}{l}\text { Right (R) } \\
\text { Left (L) }\end{array}$ & $\begin{array}{l}\text { Right sinus of Valsalva } \\
\text { Left Sinus of Valsalva }\end{array}$ \\
\hline \multirow[t]{3}{*}{ Anatomical Distribution } & 1 & Single coronary artery with normal right or left coursing ( $R C$ or $L C$ ) \\
\hline & " & $\begin{array}{l}\text { After leaving the right or left sinus the single coronary artery crosses at the } \\
\text { base of the heart as a large transverse trunk in order to supply the } \\
\text { contralateral coronary artery. }\end{array}$ \\
\hline & III & $\begin{array}{l}\text { Single coronary artery arising from the right sinus, with the LAD and the } L C X \\
\text { arteries arising from separate coronary artery trunks instead of a single trunk } \\
\text { just immediatelyat the exit. }\end{array}$ \\
\hline \multirow[t]{5}{*}{ Course of the transverse branch } & A & Anterior to the large vessels (anterior to right ventricle) \\
\hline & B & Between the aorta and pulmonary artery \\
\hline & $\mathrm{P}$ & Posterior to Large vessels \\
\hline & $\mathrm{s}$ & Septal type (above the interventricular septum) \\
\hline & $c$ & Combined type \\
\hline
\end{tabular}

\section{Conflicts of Interest: None}

\section{References}

1. Mohanty A. A review on 'single coronary artery.' Imaging Med 2015; 7: 1-2.

2. Lingaraju S, Maurya R, Sanghvi S. A study of incidence and pattern of coronary artery anomalies in Western Rajasthan, India. Int J Res Med Sci 2016.

3. Diwan Y, Diwan D, Chauhan RS, Negi PC. Coronary artery anomalies in North Indian population: a conventional coronary angiographic study. Natl J Clin Anat. 2017.

4. Lipton MJ, Barry WH, Obrez I, Silverman JF, Wexler L. Isolated single coronary artery: Diagnosis, angiographic classification, and clinical significance. Radiology 1979.

5. Yamanaka O, Hobbs RE. Coronary artery anomalies in 126,595 patients undergoing coronary arteriography. Cathet Cardiovasc Diagn. 1990;

6. Evrengul H, Ozcan EE, Turhan H, Ozturk A. Single coronary artery originating from the right sinus of Valsalva and hypoplastic left anterior descending artery: An extremely rare combination of congenital coronary artery anomalies. Exp Clin Cardiol 2012.

7. Shizukuda Y, Abo-Salem E, Helmy T. Complex single ostium coronary artery from the right coronary sinus with unique course of anomalous left circumflex coronary artery. J Cardiol Cases 2016.

8. Taylor AJ, Rogan KM, Virmani R. Sudden cardiac death associated with isolated congenital coronary artery anomalies. J Am Coll Cardiol 1992.

9. Kim JM, Lee OJ, Kang IS, Huh J, Song J, Kim G. A rare type of single coronary artery with right coronary artery originating from the left circumflex artery in a child. Korean J Pediatr 2015.

10. Panduranga $P$, Riyami AA. Single coronary artery from right aortic sinus in a very elderly patient. J Saudi Hear Assoc 2016.

11. Karahan Z, Altıntas B. Very Unusual Case Report: Type RIII of Lipton's Classification. J Cardiovasc Dis Diagnosis 2016. 